\title{
LUNG HERNIA SECONDARY TO CONGENITAL ABSENCE OF RIBS*
}

\author{
BY \\ P. P. RICKHAM \\ From Alder Hey Children's Hospital, Liverpool
}

It is usually assumed that lung hernia secondary to congenital absence of ribs is a great rarity. Peräsalo and Laustela (1956) describing the latest published case, of a man aged 28 years, could not find any reference to this deformity in the literature and only one of the recently published books on paediatric surgery (Swenson, 1958) mentions the malformation.

Perusal of the literature, however, does indicate that lung herniae are not so very uncommon. The earliest reported case is that by Rolland, writing in Guy de Chauliac's 'System of Surgery', in 1499. All early reports concerned lung hernias secondary to trauma or disease of the thoracic wall. The first congenital case to be reported was that of MorelLavallée in 1847. By 1933 Goodman was able to collect 172 cases of lung hernias of all types from the literature; about $18 \%$ of these were congenital.

In practically all the congenital herniae of the lung reported in the literature the defect in the thoracic wall was small. In some the defect was due to absence of the para-sternal ends of one or two ribs; lung hernia secondary to complete absence of ribs is uncommon. Absent ribs are, of course, frequently encountered and are often associated with spinal deformities, but it is usually the first, second, eleventh or twelfth ribs which are absent, and absence of the most proximal or distal ribs does not commonly produce lung hernia. Few of the cases reported in the literature seem to have given rise to important symptoms during early infancy. The bulging hernia and paradoxical respiration were noticed, but the infants do not seem to have been unduly distressed. In later life, however, respiratory distress, cough, recurrent bronchitis and pain are common symptoms and have often necessitated operative correction.

Various methods have been used in the treatment of congenital lung hernia. Tight bandaging of the chest wall does not appear to have been any good and has occasionally produced marked distress.

\footnotetext{
- A paper read at a meeting of the British Association of Paediatric Surgeons held in London in July, 1958.
}

Fascia lata grafts (D'Abreu, 1953) are only practicable for small defects and unsuitable for growing infants. Bone graft operations have been performed, either free bone grafts from other parts of the skeleton (Peräsalo and Laustela, 1956) or from adjacent ribs (Montgomery and Lutz, 1925).

At the time when the case described below was operated upon we were unaware of the methods of treatment reported in the literature.

\section{Case Report}

K.A., a baby girl, was born on April 18, 1957. An enormous lung hernia was easily seen and the child suffered immediate acute respiratory distress and needed to be continuously nursed in oxygen. Even in oxygen the infant had repeated severe attacks of cyanosis, and became very distressed whilst feeding. During the succeeding weeks she improved greatly and it was possible to nurse her out of oxygen, but she had recurrent attacks of bronchopneumonia, did not gain weight and became cyanosed and distressed when feeding or crying.

She was referred to Alder Hey Children's Hospital when 7 weeks old. On examination the child's state of nutrition was poor. There was a very large bulge over the left side of the thorax, which was tympanic and showed paradoxical respiratory movements. These movements were exaggerated when the child cried and she became cyanosed when crying. Clinically the patient was found to have complete absence of the left sixth, seventh, eighth, ninth and tenth ribs; this was confirmed radiologically. There was a marked right thoracic scoliosis.

Radiography of the chest confirmed the absence of five ribs of the left side, and showed the fifth rib to be broader than normal. The associated spinal scoliosis was due to wedging of the sixth to tenth thoracic vertebrae The clinical and radiographic defects are shown on p. 16 .

Because of the child's failure to thrive and her repeated attacks of bronchopneumonia it was decided to attempt surgical correction of the deformity. At operation under endotracheal nitrous oxide and oxygen anaesthesia, with continuous intravenous succinyl-choline administration and controlled respiration, the thoracic wall defect was exposed through an oblique incision across the maximum convexity of the bulge. The fifth, eleventh and twelfth 


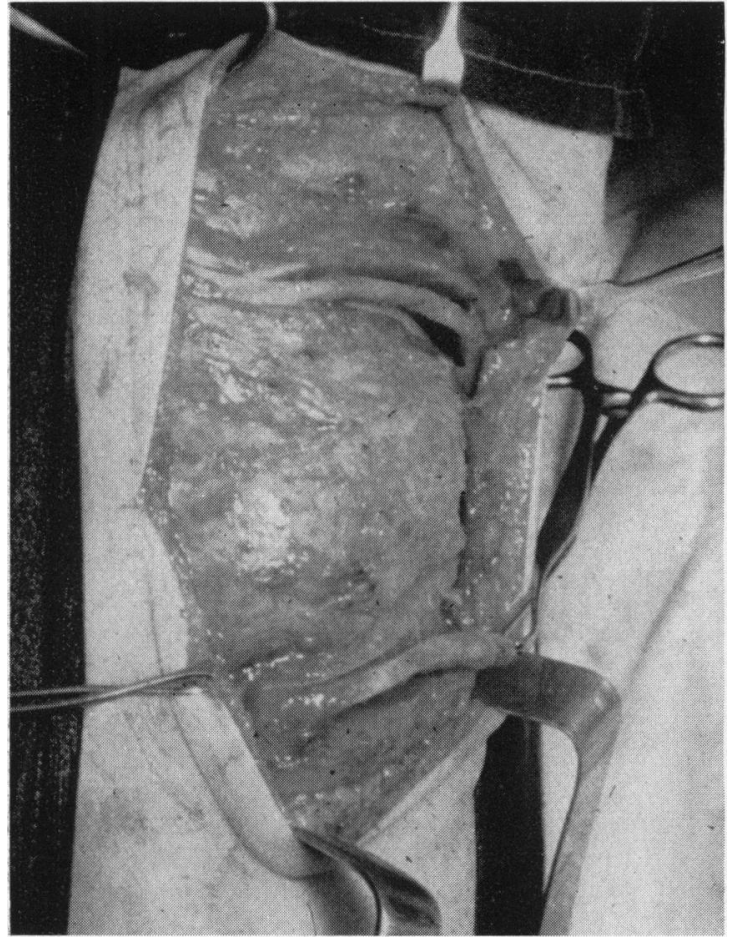

FIG. 1.-The 5th, 11 th and 12 th ribs are exposed.

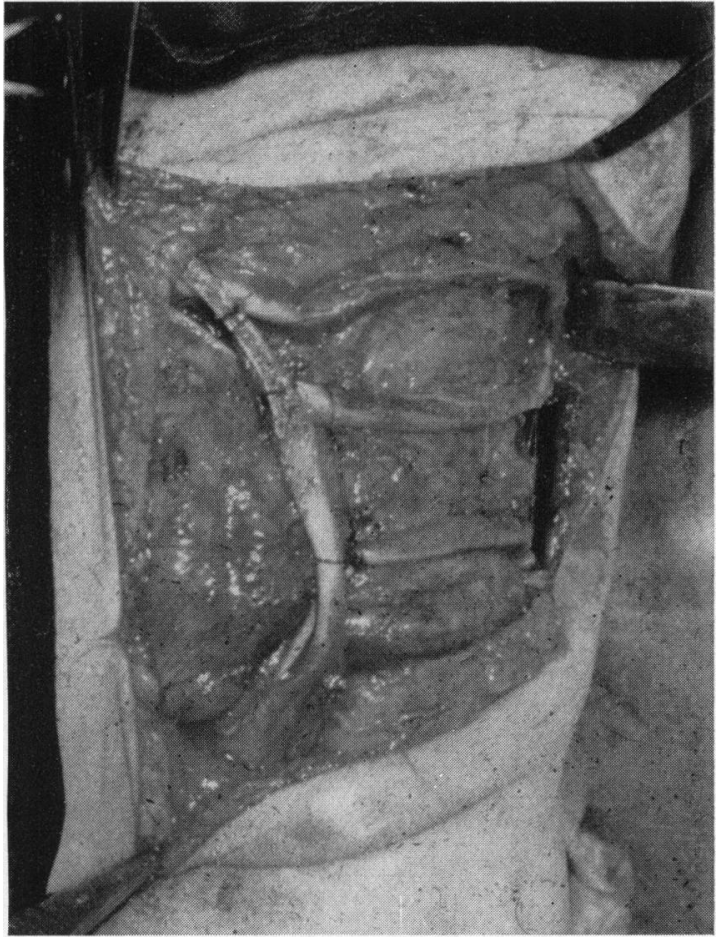

FIG. 2.-The rib grafts have been fixed to each other.

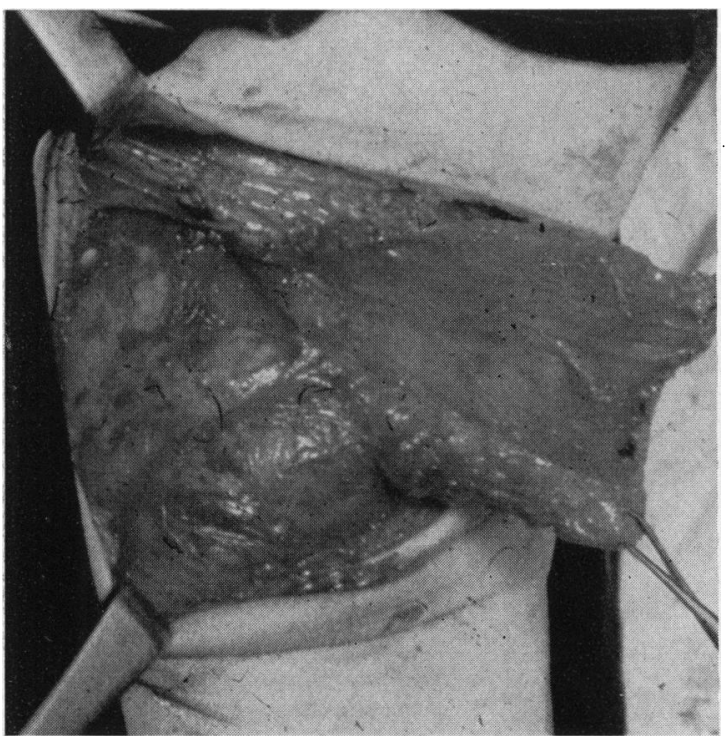

Fig. 3.-The external oblique muscle is elevated after dividing its insertion into the rectus sheath.

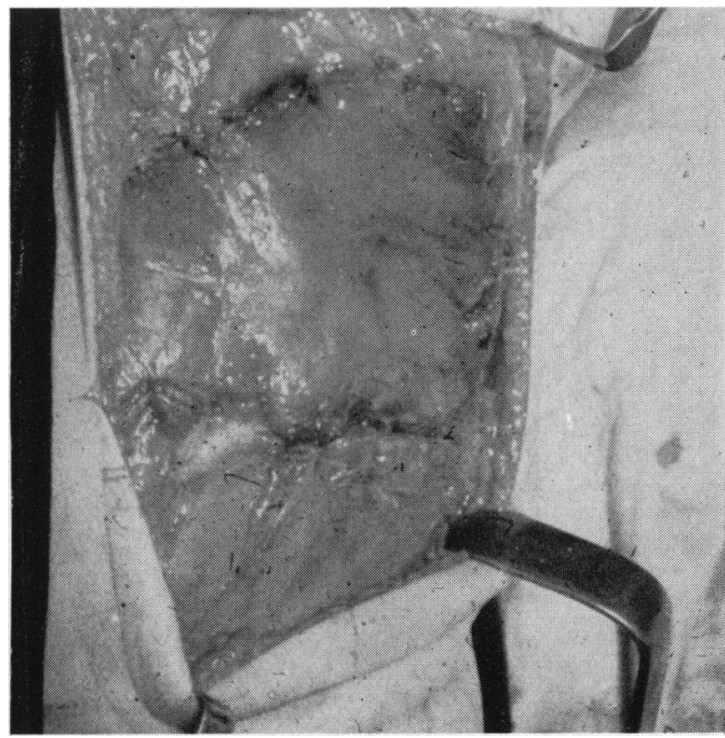

Fig. 4.-The external oblique muscle has been flapped backwards and is sewn across the defect. 


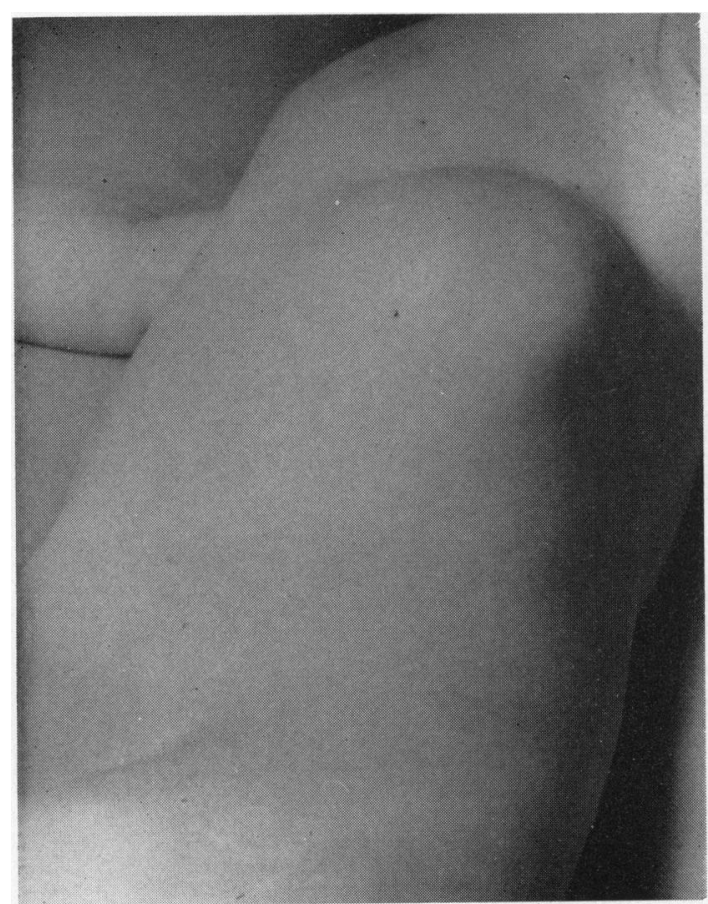

FIG. 5.-The bulging lung hernia.

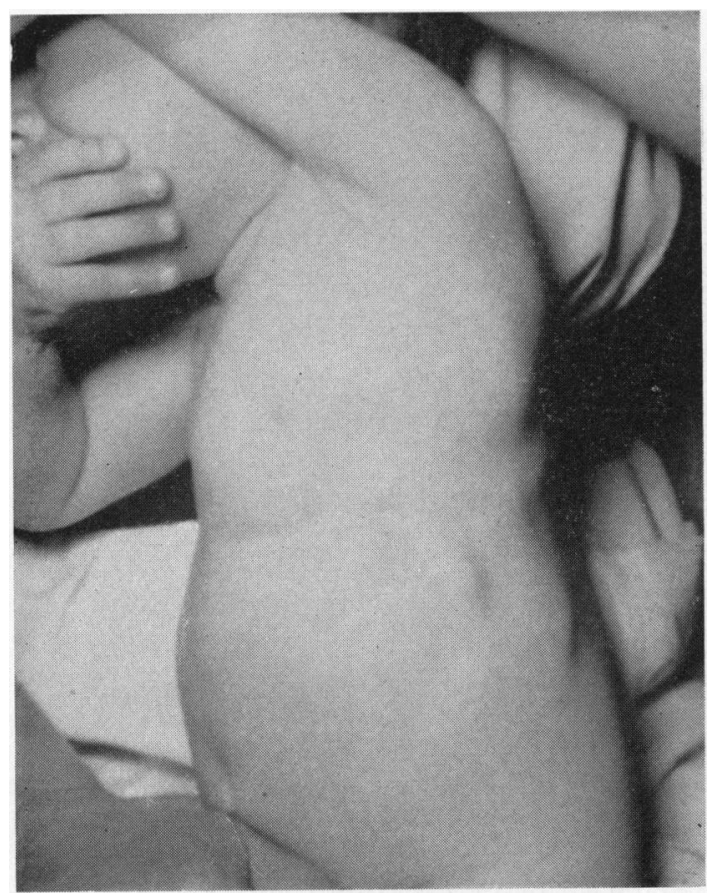

FIG. 7.-Post-operative appearance, one year after operation.

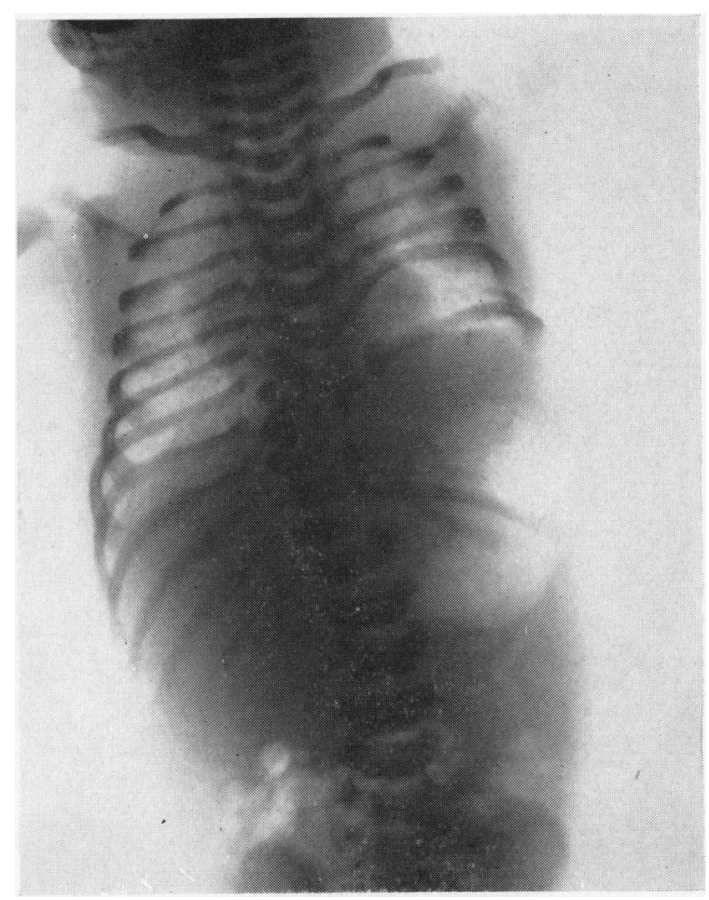

FIG. 6.-Pre-operative radiograph.

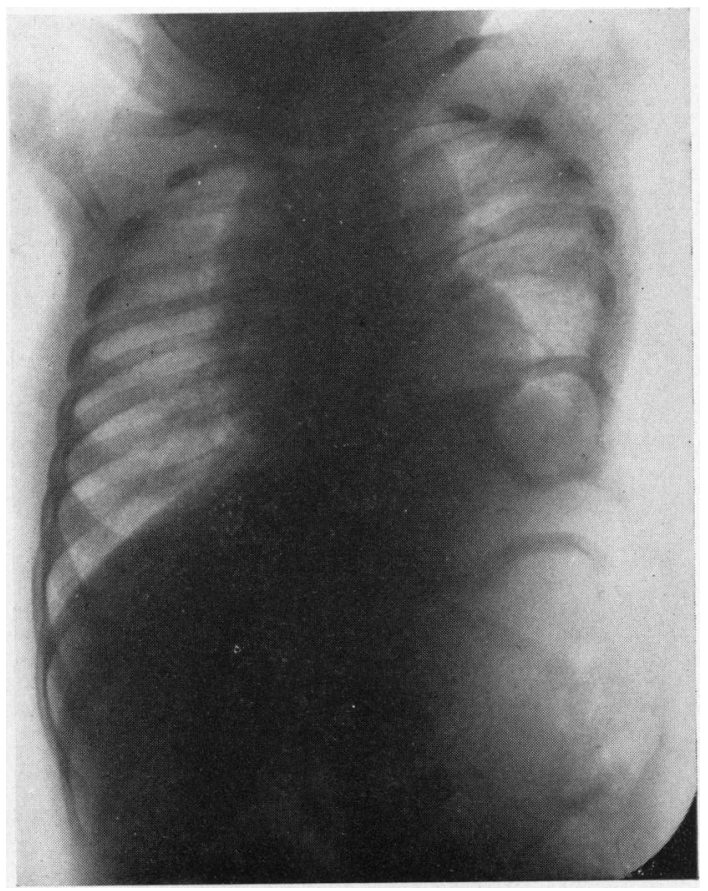

FIG. 8.-Post-operative radiograph one year after operation. 
ribs were exposed (Fig. 1). Because of the great size of the defect and the youth of the patient it was decided not to attempt repair with a free fascia lata graft nor to employ any plastic materials. The fifth rib was split longitudinally with a fine saw, taking care that the periostium remained attached to both halves of the rib. The extreme anterior and posterior ends of the rib were not divided. With the aid of pericostal nylon sutures it was possible to pull the lower half of the fifth rib downwards towards the eleventh rib. The eleventh rib was similarly split, but this time the posterior end of the the upper half of the rib was divided, swung around its anterior attachment and stitched to the anterior extremity of the fifth rib thus forming a costal margin. Because of the shortness of the eleventh rib this artificial costal margin was, however, situated more posteriorly than the costal margin on the opposite side (Fig. 2). The whole insertion of the left external oblique muscle to the rectus sheath was then divided, the muscle was dissected out and reflected backwards across the whole of the thoracic wall defect (Fig. 3). It was then stitched to the surrounding muscles (Fig. 4). The wound was closed without drainage.

The child stood the operation well and there was no respiratory difficulty during the post-operative period. Since then she has developed satisfactorily and there are now no paradoxical movements on the left side of the chest. The posterior displacement of the left costal margin is, however, noticeable, and is accentuated by the thoracic scoliosis. Radiography of the chest one year afterjoperation shows that the rib grafts have survived.
Clinically the child's condition is very satisfactory. The clinical and radiographic appearances before and after operation are shown in Figs. 5, 6, 7 and 8.

There is little doubt that lung hernia secondary to congenital absence of ribs is more common than is realized. Extensive defects like the one described in this paper appear, however, to be uncommon and as they are liable to endanger the child's life during infancy, operative correction is advisable. The operative procedure reported here has produced a gratifying result.

\section{Summary}

A case of extensive pulmonary hernia secondary to congenital absence of five ribs is reported, and the surgical correction of the malformation is described. The literature is briefly reviewed.

I should like to thank Dr. Ann McCandless who referred the case to me, and Dr. G. Jackson Rees for the skilfully administered anaesthetic.

REFERENCES

D'Abreu, A. L. (1953). A Practice of Thoracic Surgery. Arnold, London:

Goodman, H. I. (1933). J. thorac. Surg., 2, 368.

Montgomery, J. G. and Lutz, H. (1925). Ann. Surg., 82, 22.

Morel-Lavallée, J. (1847). Mém. Soc. Chir., Paris, 1, 75.

Peräsalo, $O$, and Laustela, E. (1956). Ann. Chir. Gynaec. Fenn., 45, 303.

Rolland (1499). In Guy de Chauliac's System of Surgery.

Rolland (1499). In Guy de Chauliac's System of Surgery. Inc., New York. 